\title{
Numerical Simulation of Incompressible Flows around a Fish Model at Low Reynolds Number Using Seamless Virtual Boundary Method"
}

\author{
Hidetoshi NISHIDA $^{* *}$ and Kyohei TAJIRI ${ }^{* *}$ \\ **Department of Mechanical and System Engineering, Graduate School of Science and Technology, \\ Kyoto Institute of Technology, \\ Matsugasaki, Sakyo-ku, Kyoto 606-8585, Japan \\ E-mail: nishida@kit.ac.jp
}

\begin{abstract}
In this paper, the numerical simulation of incompressible flows around a fish model at low Reynolds number using the seamless virtual boundary method is presented. In order to satisfy the velocity conditions on the virtual boundary points, the forcing term is added not only on the grid points near the boundary but also on the grid points inside the boundary in the seamless virtual boundary method, so that the smooth physical quantities can be obtained. The present approach is verified by the flows around a 2D oscillating circular cylinder and a sphere. The flows around the stationary and swimming fish models are simulated for application to the more complicated flow geometry with moving boundary. These results show that the flows with the stationary and moving bodies can be predicted precisely. Then, it is concluded that the present seamless virtual boundary method is very promising for the numerical simulation of incompressible flows with the complicated geometry and the moving boundary.
\end{abstract}

Key words: CFD, Cartesian Approach, Seamless Virtual Boundary Method, Moving Boundary, Incompressible Flow

\section{Introduction}

For the practical flow simulations, the boundary fitted coordinates (BFC) is usually adopted. This BFC approach has the high adaptability to the boundary configuration. However, in the complicated flow geometry, it is difficult to generate the computational grid. Moreover, in the BFC approach, it is necessary that the governing equations are transformed from the physical plane to the computational plane, so that the transformed governing equations have more terms than the original equations in the Cartesian coordinates. Therefore, the computational effort is larger than the Cartesian grid approach. Then, in recent years, the Cartesian grid approach is highlighted again for the numerical flow simulations.

In the Cartesian grid approaches, especially, the immersed boundary method is applied to many simulations of incompressible flow. One of this immersed boundary method is the virtual boundary method ${ }^{(1)-(4)}$. In order to satisfy the velocity conditions on the (virtual) boundary points, the virtual boundary method requires the external forcing term added to the momentum equations. This forcing term is usually estimated by two ways, i.e., feedback ${ }^{(2)-(4)}$ and direct ${ }^{(1)}$ procedures. Also, in both forcing term estimations, the grid points added the forcing term are restricted only near the boundary. Therefore, the unphysical oscillations near the boundary appear in the pressure field. In order to improve this weak 
point, Tyagi and Acharya ${ }^{(5)}$ introduced the forcing terms on both sides of the immersed boundary, i.e., two-side forcing scheme. Ye et al. ${ }^{(6)}$ proposed the reshaped cell method in which the spatial derivatives of the governing equations were discretized in cell that was cut by the boundaries. Tseng and Ferziger ${ }^{(7)}$ developed the immersed boundary method with second order ghost cell reconstruction to enforce the boundary conditions. Kim et al. ${ }^{(8)}$ added the mass source/sink term to the continuity equation in order to satisfy the mass conservation at cells containing the immersed boundary. Nishida and Sasao ${ }^{(9)}$ proposed the seamless virtual boundary method. In this method, the forcing term is added not only on the grid points near the boundary but also in the region inside the boundary, so that the unphysical pressure oscillations are removed.

In this paper, we try to apply the seamless virtual boundary method to the complicated flow simulation with moving boundary. First, in order to validate the present seamless virtual boundary method, the numerical simulations of incompressible flows around a 2D oscillating circular cylinder and a sphere are considered. Finally, the numerical simulations of flow around the stationary and swimming fish models are carried out. The effect of fins, i.e., the breast fins, abdomen fins, hip fin, back fin and tail fin, is discussed.

\section{Seamless Virtual Boundary Method}

\subsection{Governing equations}

In the virtual boundary method, the velocity conditions on the virtual boundary are satisfied by adding the forcing term to the momentum equations. Then, the incompressible Navier-Stokes equations can be written in non-dimensional form by

$$
\begin{aligned}
& \frac{\partial u_{i}}{\partial x_{i}}=0 \\
& \frac{\partial u_{i}}{\partial t}+u_{j} \frac{\partial u_{i}}{\partial x_{j}}=-\frac{\partial p}{\partial x_{i}}+\frac{1}{R e} \frac{\partial^{2} u_{i}}{\partial x_{j} \partial x_{j}}+G_{i},
\end{aligned}
$$

where $R e$ denotes the Reynolds number defined by $R e=U L / v . U, L$ and $v$ are the reference velocity, the reference length and the kinematic viscosity, respectively. The last term in Eq.(2), $G_{i}$, denotes the additional forcing term.

\subsection{Forcing term estimation}

In order to estimate the additional forcing term in the governing equations, $G_{i}$, there are mainly two ways, that is, the feedback ${ }^{(2)-(4)}$ and direct $^{(1)}$ forcing term estimations. In this paper, the direct forcing term estimation shown in Fig.1 is adopted. We explain in 2D but the extension to $3 \mathrm{D}$ is straightforward.

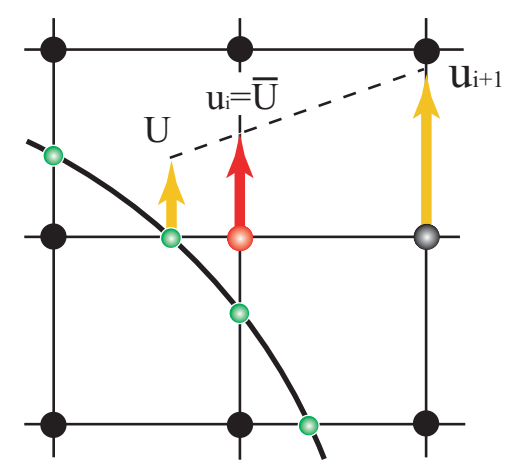

Fig. 1 Direct forcing terms estimation. 
For the forward Euler time integration, the forcing term can be determined by

$$
G_{i}=\left[u_{j} \frac{\partial u_{i}}{\partial x_{j}}+\frac{\partial p}{\partial x_{i}}-\frac{1}{R e} \frac{\partial^{2} u_{i}}{\partial x_{j} \partial x_{j}}\right]^{n}+\frac{\bar{U}_{i}^{n+1}-u_{i}^{n}}{\Delta t}
$$

where $\bar{U}_{i}^{n+1}$ denotes the interpolated velocity by linear interpolation. Namely, the external force is specified as the velocity components at next time step satisfy the relation, $u_{i}^{n+1}=\bar{U}_{i}^{n+1}$. In this forcing term estimation, the grid points added forcing term are restricted near the boundary. Then, the pressure distributions near the boundary show unphysical oscillations.

\subsection{Seamless virtual boundary method}

In order to remove aforementioned unphysical oscillations near the boundary, the seamless virtual boundary method ${ }^{(9)}$ was proposed. In the seamless virtual boundary method, the forcing term is added not only near the boundary but also in the region inside the boundary shown in Fig.2. On the grid points near the boundary, the additional forcing term is estimated by the same procedure as the usual direct forcing term estimation, Eq.(3). In the region inside the boundary, the forcing term is determined by satisfying the relation, $\bar{U}_{i}^{n+1}=U_{b}$, where $U_{b}$ is the specified velocity, e.g., $U_{b}=0$ in stationary solid media.

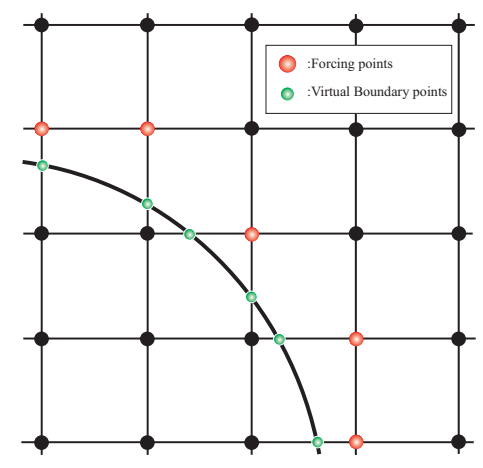

(a) Usual virtual boundary method.

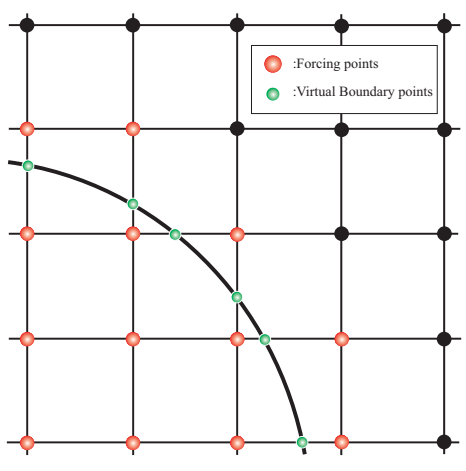

(b) Seamless virtual boundary method.

Fig. 2 Grid points added forcing terms.

\subsection{Numerical method}

The incompressible Navier-Stokes equations (2) are solved by the second order finite difference method on the collocated grid arrangement. The convective terms are discretized by the second order fully conservative finite difference method ${ }^{(10)}$. The diffusion and pressure terms are discretized by the usual second order centered finite difference method. For the time integration, the fractional step approach ${ }^{(11)}$ based on the two-step Runge-Kutta scheme $^{(12)}$ is applied. For the usual incompressible Navier-Stokes equations, the two-step Runge-Kutta scheme is written by

$$
u_{i}^{n+1}=(1-\gamma) u_{i}^{n}+\gamma u_{i}^{n-1}+\Delta t\left[\alpha\left(F_{i}^{n}-\nabla p^{n}\right)+\beta\left(F_{i}^{n-1}-\nabla p^{n-1}\right)\right],
$$

where $\alpha, \beta$, and $\gamma$ are the parameters of two-step Runge-Kutta scheme and $F_{i}$ denotes the convective and diffusion terms. In this paper, the parameters are set as $\alpha=9 / 8, \beta=-7 / 8$, $\gamma=-3 / 4$ in order to satisfy the second order of time accuracy. The fractional step approach based on the two-step Runge-Kutta scheme can be written by 


$$
\begin{aligned}
& u_{i}^{*}=(1-\gamma) u_{i}^{n}+\gamma u_{i}^{n-1}+\Delta t\left[\alpha F_{i}^{n}+\beta\left(F_{i}^{n-1}-\nabla p^{n-1}\right)\right], \\
& u_{i}^{n+1}=u_{i}^{*}-\Delta t\left(\alpha \nabla p^{n}-G_{i}\right),
\end{aligned}
$$

where $u_{i}{ }^{*}$ denotes the fractional step velocity. The resulting pressure equation is solved by the SOR method. The convergence criterion of the momentum equations and pressure equation is set as $\phi_{L 2}<1.0 \times 10^{-6}$, where $\phi_{L 2}$ is the L-2 residual of physical quantities $\phi$, i.e., the velocity or pressure.

\section{Validation of Seamless Virtual Boundary Method}

\subsection{Flow around a 2D oscillating circular cylinder}

The computational domain is shown in Fig.3. The circular cylinder in the uniform flow moves vertically as,

$$
y(t)=y_{0}+\frac{y_{a m p}}{2} \sin (2 \pi f t)
$$

where the amplitude is $y_{a m p} / 2=0.2 D$ and the non-dimensional frequency is $f=0.2$. The initial location of circular cylinder is $\left(x_{0}, y_{0}\right)=(5.5 D, 5.5 D)$. The grid resolution is $293 \times 253$ with non-uniform grid system. The impulsive start determined by the uniform flow is adopted. On the inflow boundary, the velocity is fixed by the uniform flow and the pressure is imposed by the Neumann condition obtained by the normal momentum equation. The velocity is extrapolated from the inner points and the pressure is obtained by the Sommerfeld radiation condition ${ }^{(13)}$ on the outflow and side boundaries. On the virtual boundary and inside the boundary, the moving circular cylinder velocity is imposed.

Figure 4 shows the pressure contours with $R e=200$ at top and bottom locations. These results show that the vortex shedding becomes larger than the flow around a stationary circular cylinder. In the close-up views, the smooth pressure fields near the circular cylinder can be obtained. The present drag and lift coefficients and the Strouhal number shown in Table 1 are in very good agreement with the reference result ${ }^{(14)}$.

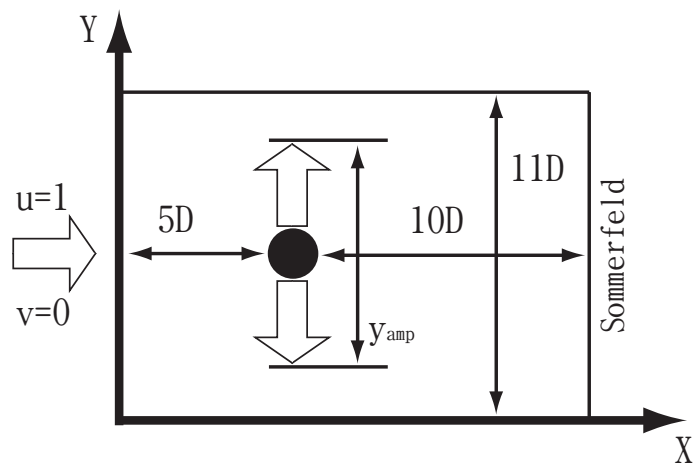

Fig. 3 Computational domain.

Table 1 Comparison of flow characteristics.

\begin{tabular}{|l|c|c|c|}
\hline & $C_{D}$ & $C_{L}$ & $S t$ \\
\hline Present & $1.59 \pm 0.17$ & \pm 0.46 & 0.20 \\
\hline Wu et al. ${ }^{(14)}$ & $1.58 \pm 0.20$ & \pm 0.58 & 0.20 \\
\hline
\end{tabular}




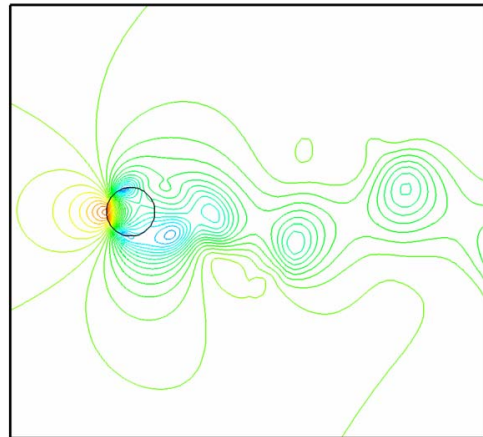

(a) Overall view $(\mathrm{t}=211.25)$.

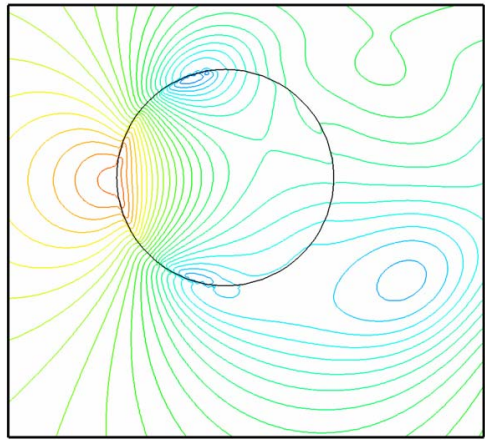

(c) Close-up view ( $\mathrm{t}=211.25)$.

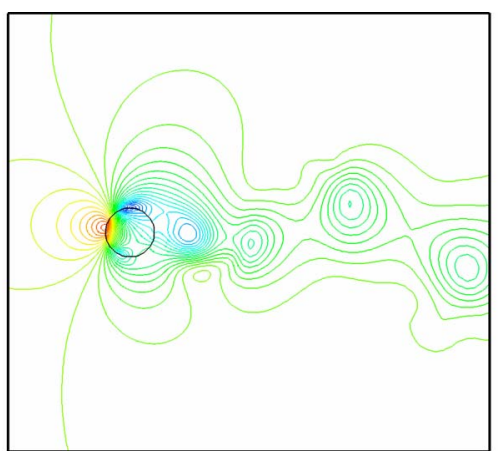

(b) Overall view $(\mathrm{t}=215)$.

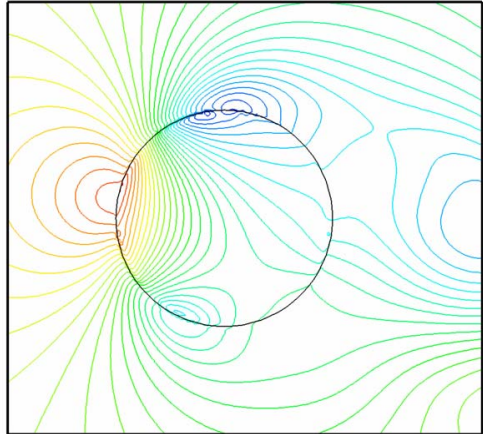

(d) Close-up view $(\mathrm{t}=215)$.

Fig. 4 Pressure contours.

\subsection{Flow around a sphere}

Figure 5 shows the computational domain. The initial and boundary conditions are the same as the previous simulation. On the virtual boundary and inside the boundary, the non-slip velocity condition $\left(u_{i}=0\right)$ is imposed. In order to reduce the number of grid points, the hierarchical Cartesian grid with 5 levels is introduced. The body shape is determined by the triangular polygon, so that the arbitrary body surface can be represented. The sphere surface generated by the 4900 triangular polygons is shown in Fig.6. The steady flow $(R e=100)$ and unsteady flow $(R e=300)$ are simulated with about1.8 million grid points.

In Fig. 7 the pressure contours with $R e=100$ at the steady state is shown. The unphysical oscillations do not appear, so that the smooth pressure field can be obtained. Also, the symmetrical flow field can be reappeared. Figure 8 shows the iso-surface of second invariant of velocity gradient tensor $Q^{\prime}$ with $R e=300$. The characteristic periodical hairpin vortex is observed clearly. Table 2 shows the drag coefficient and vortex length in comparison with the reference solution ${ }^{(15)}$. It is confirmed that the appropriate solutions can be obtained. Then, the present approach gives the accurate solutions in the $3 \mathrm{D}$ simulation.

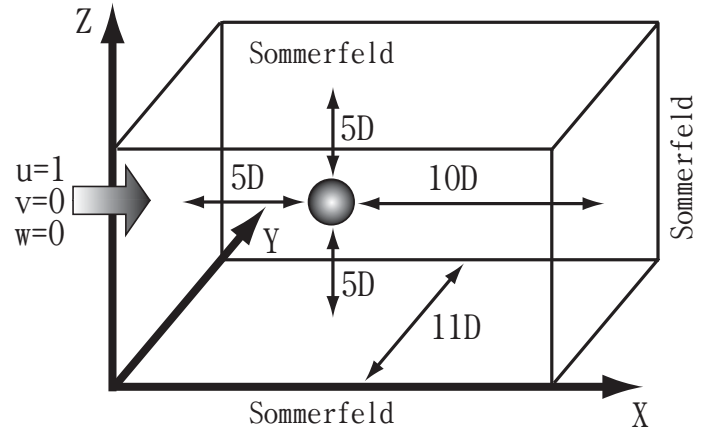

Fig. 5 Computational domain.

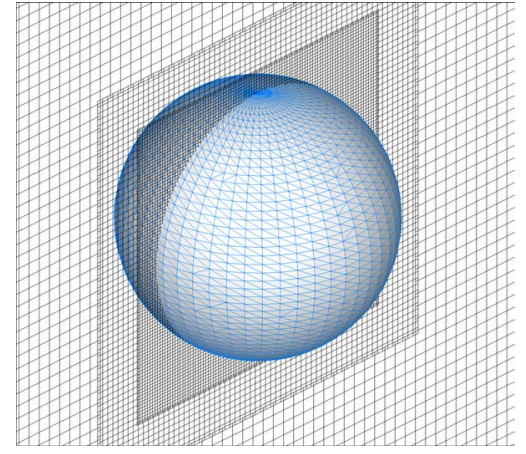

Fig. 6 Surface mesh and hierarchical grid. 


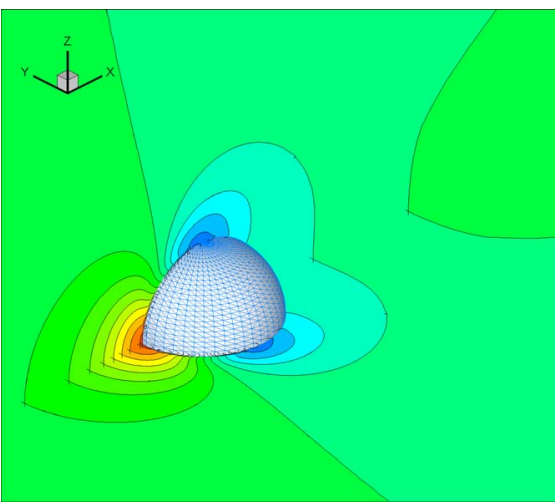

Table 2 Comparison of flow characteristics $(\mathrm{Re}=100)$.

Fig. 7 Pressure contours.

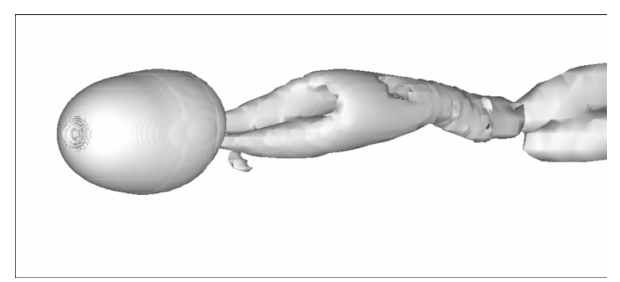

(a) $\mathrm{t}=122$.

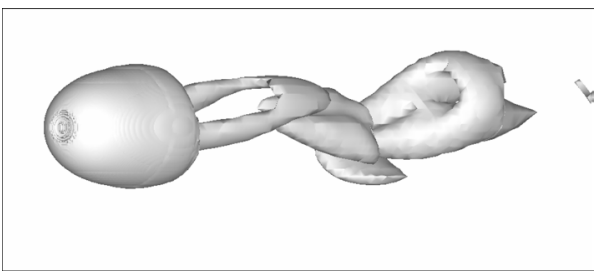

(c) $\mathrm{t}=126$.

\begin{tabular}{|l|c|c|}
\hline & $C_{D}$ & $L_{V} / D$ \\
\hline Present & 1.109 & 0.867 \\
\hline Shirayama $^{(15)}$ & 1.104 & 0.844 \\
\hline
\end{tabular}

Fig. 8 Iso-surface of second invariant of velocity gradient tensor.

\section{Application to Flow around a Fish Model}

\subsection{Stationary fish model}

In this paper, we consider the acheilognathus moriokae (Japanese bitterling) in the uniform flow. The surface shape generated by the triangular polygon is shown in Fig.9. The reference length $D$ is body length of a fish and the total number of triangular polygons is 5866. The characteristic shape of a fish, i.e., the breast fins, abdomen fins, hip fin, back fin and tail fin, can be reappeared. Figures 10 and 11 show the computational domain and the used 5 leveled hierarchical grid with about 3.8 million grid points.

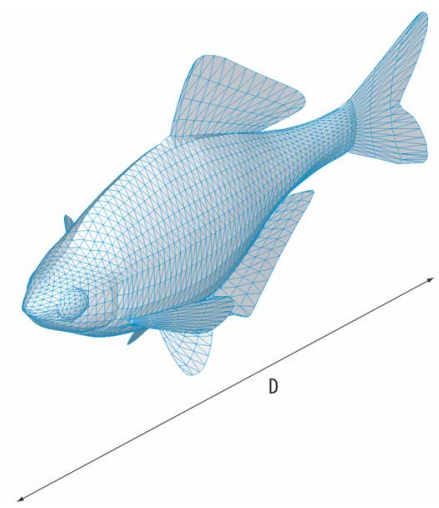

Fig. 9 Surface shape of a fish model. 


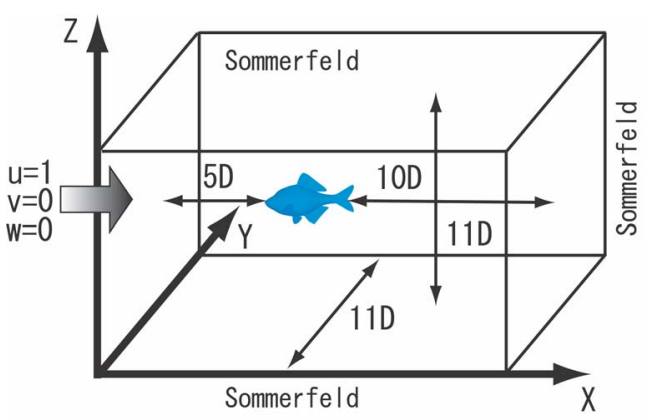

Fig. 10 Computational domain.

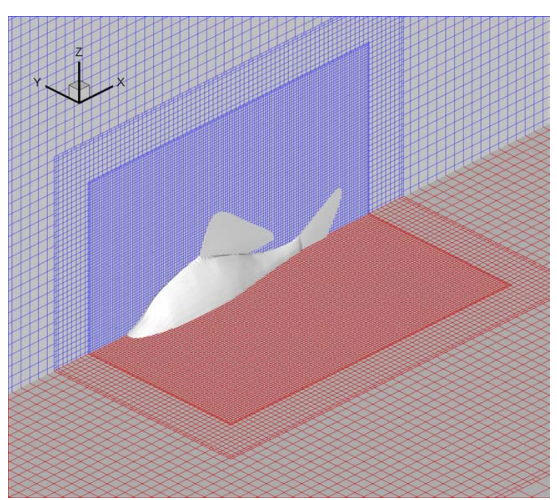

Fig. 11 Hierarchical grid with 5 levels.

The initial and boundary conditions are the same as the flow around a sphere. On the virtual boundary and inside the boundary, the non-slip velocity condition $\left(u_{i}=0\right)$ is imposed. The simulation is carried out for steady flow with $R e=100$.

The pressure contours and its drawing plane are shown in Fig.12. The smooth pressure distributions can be obtained in any plane. In $x-y$ plane, the pressure field represents the symmetric distribution comparable to the model symmetry. Also, the pressure distribution in the detail, i.e., around the breast fins, abdomen fins, hip fin and back fin, can be observed clearly. In this case, the non-dimensional drag force is 0.056 . The flow rate error is $0.024 \%$, so that the reasonable solution can be obtained qualitatively.

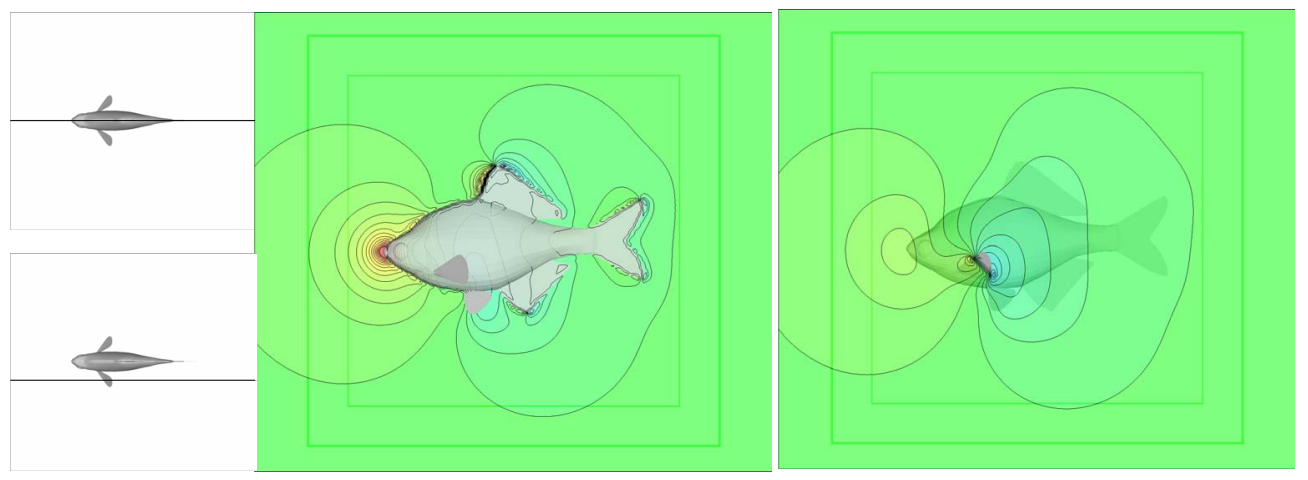

(a) Drawing plane.

(b) Pressure contours on $y=5.5 D$ and $y=5.35 D$.

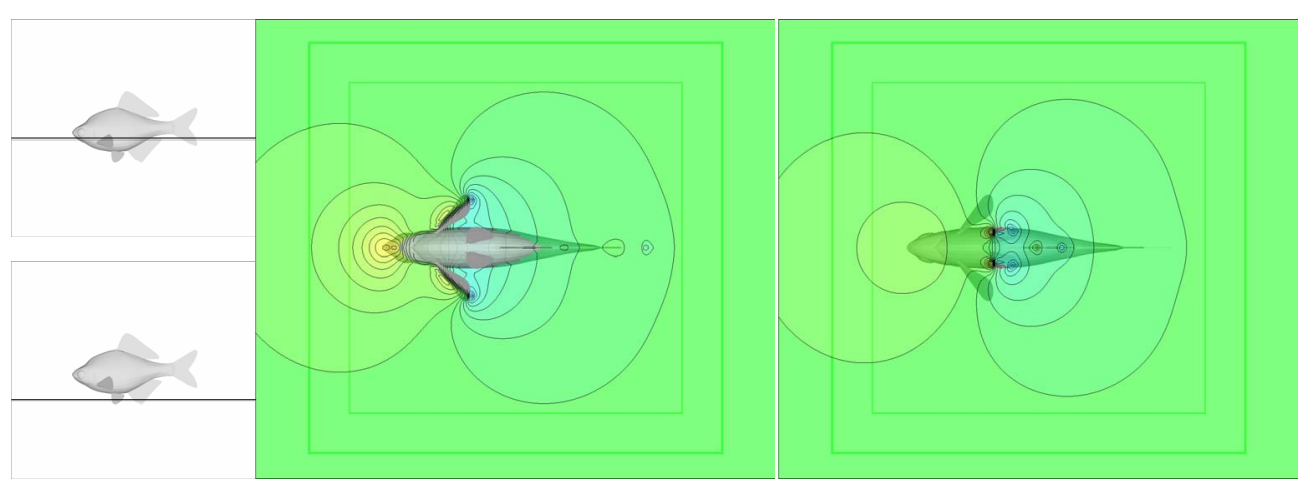

(c) Drawing plane.

(d) Pressure contours on $z=5.4 D$ and $z=5.25 D$.

Fig. 12 Pressure distributions. 


\subsection{Swimming fish model}

In this section, the swimming fish based on the previous stationary model is controlled by $2 \mathrm{D}$ rotation on four rotating axes $(S 1, S 2, S 3, S 4)$ shown in Fig.13, in order to model the practical swimming fish. $S 1$ is fixed at $(x, y)=(5.2 D, 5.5 D)$. The rotating angle on each rotating axis is defined by

$$
\begin{aligned}
& \theta 1=-\psi 1 \sin \left(2 \pi n_{f} / N_{f}\right), \\
& \theta 2=\psi 2 \sin \left(2 \pi n_{f} / N_{f}\right), \\
& \theta 3=\psi 3 \sin \left(2 \pi n_{f} / N_{f}\right), \\
& \theta 4=\psi 4 \sin \left(2 \pi n_{f} / N_{f}-\pi / 2\right),
\end{aligned}
$$

where $n_{f}$ is the frame number and $N_{f}$ denotes the total number of frames $\left(N_{f}=100\right)$. $\psi i$ is the maximum rotating angle, i.e., $\psi 1=\pi / 90, \psi 2=2 \pi / 45, \psi 3=\pi / 18$, and $\psi 4=\pi / 12$ in this paper. The swimming behavior is shown in Fig.14.

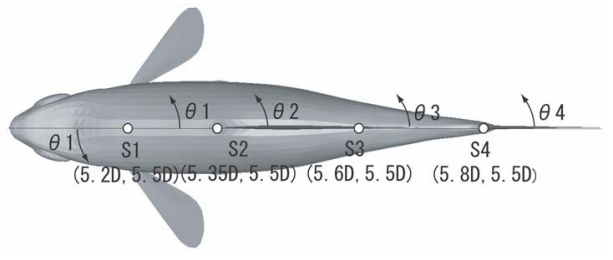

Fig. 13 Fish shape and rotating axis.
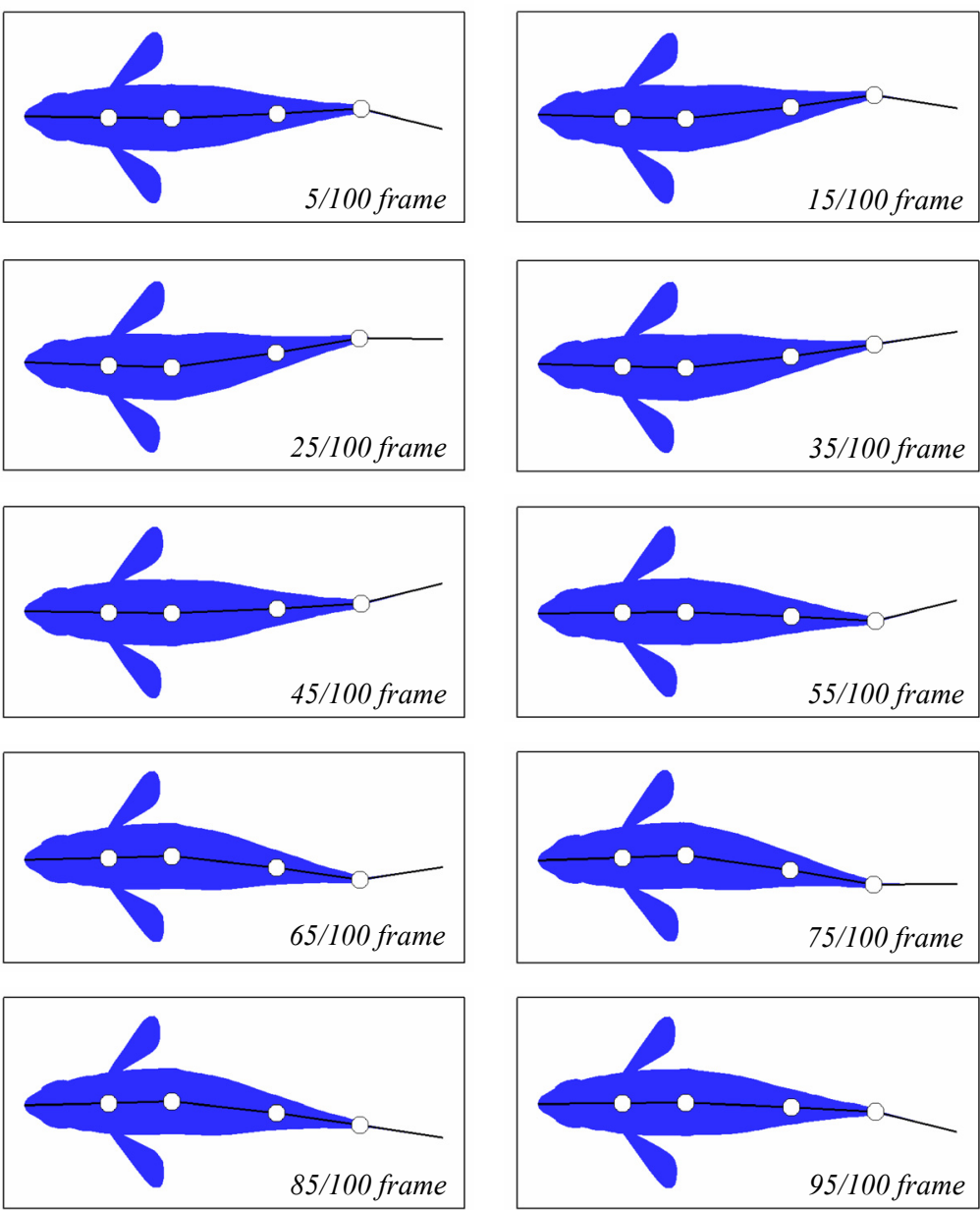

Fig. 14 Fish shape for swimming model. 


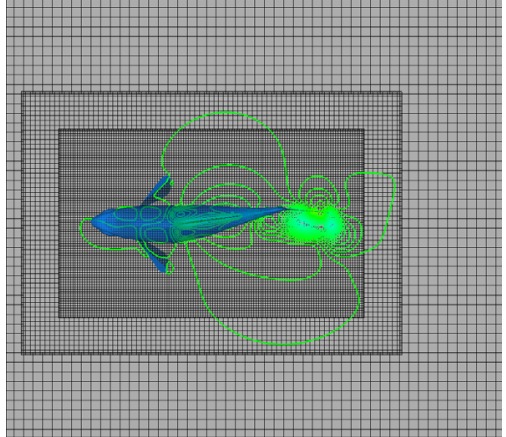

(a) $20 / 100$ frame.

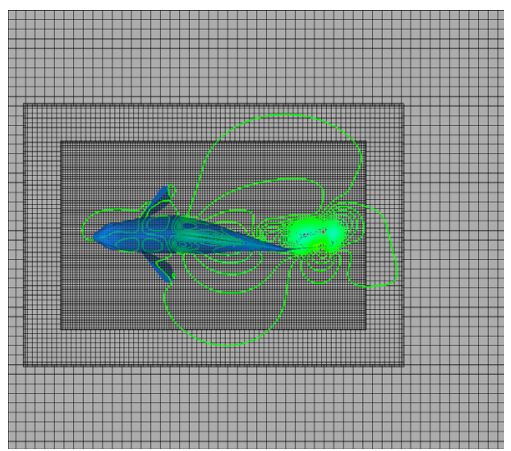

(c) 70/100 frame

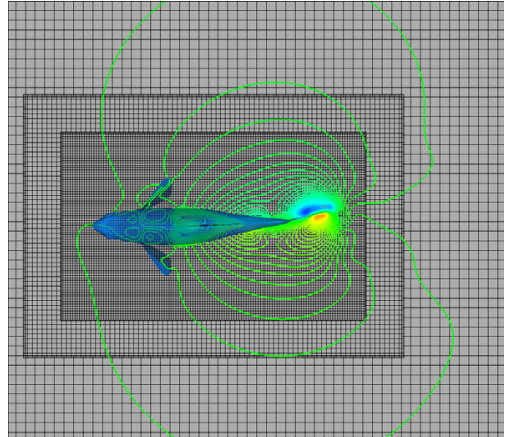

(b) $45 / 100$ frame.

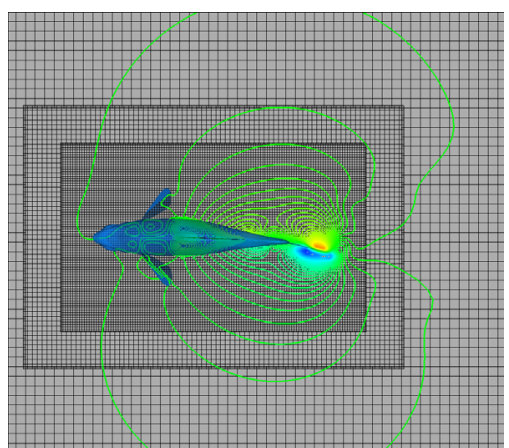

(d) $95 / 100$ frame.

Fig. 15 Pressure distributions on $x-y$ plane.

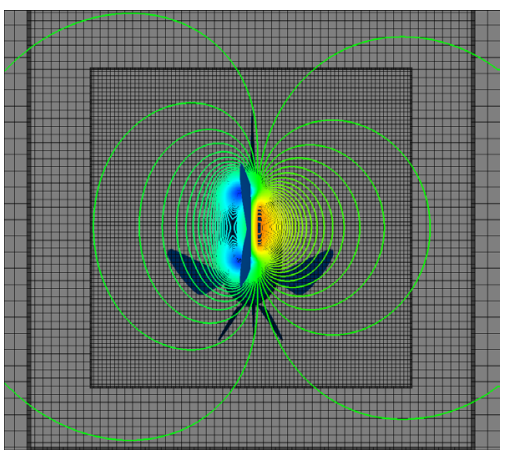

(a) $5 / 100$ frame.

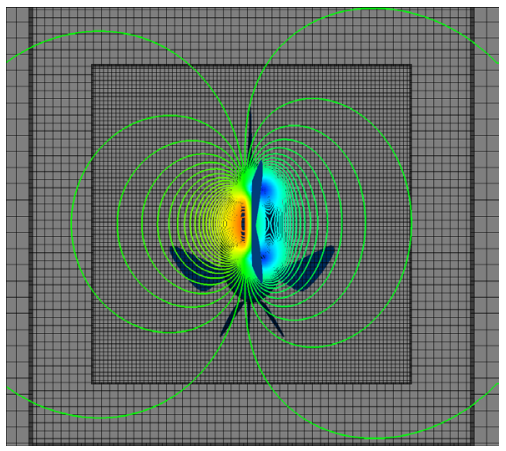

(c) 55/100 frame.

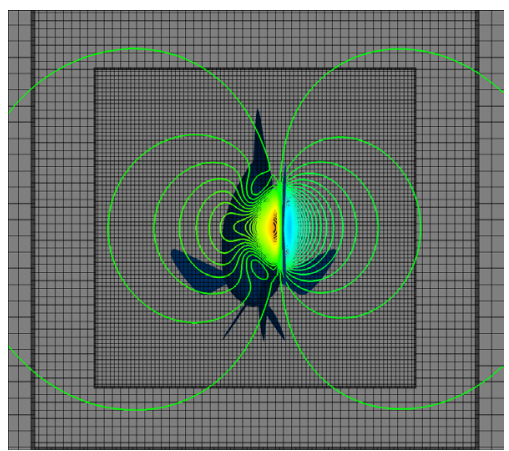

(b) $30 / 100$ frame.

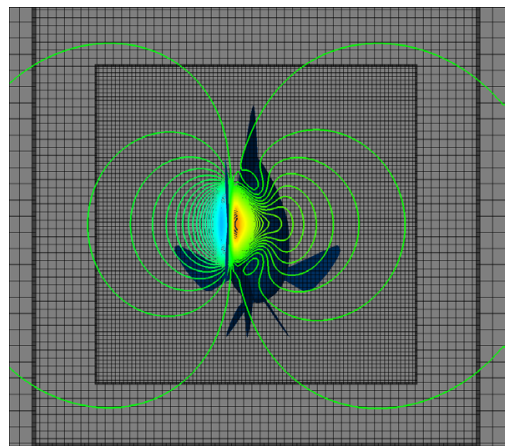

(d) $80 / 100$ frame.

Fig. 16 Pressure distributions on $y$-z plane. 
The initial and boundary conditions are the same as the stationary case. On the virtual boundary and inside the boundary, the moving velocity condition $\left(u_{i}=\left.u_{i}\right|_{\text {move }}\right)$ is imposed. $\left.u_{i}\right|_{\text {move }}$ is the moving velocity determined by Eq.(8).

The simulation with $R e=100$ is executed. Figures 15 and 16 show the pressure field with the motion in one period. Figure 15 is the pressure on $x-y$ plane $(z=5.5 D)$, and Fig. 16 is one on $y$ - $z$ plane $(x=6.0 D)$. At the tail fin, it can be confirmed that the high pressure region appears in the motion direction side and the low pressure region appears in the motion direction reverse side. The second invariant of velocity gradient tensor $Q^{\prime}$ near a swimming fish is shown in Fig.17. In this swimming model, the breast fins are always open, so that it is remarkable to form the large eddy. The comparatively small eddy with the motion of the abdomen, hip and back fins can be observed. The separation vortices from the breast, abdomen, hip and back fins are greatly shoved by the tail fin, so that the complicated vortex structures are generated near the tail fin. However, these vortices near the tail fin are transported rear and the large eddy is formed, because of the effect of large viscosity, i.e., low Reynolds number $(R e=100)$.

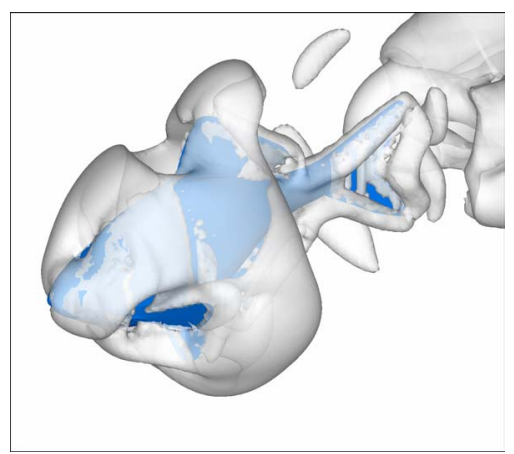

(a) 5/100 frame.

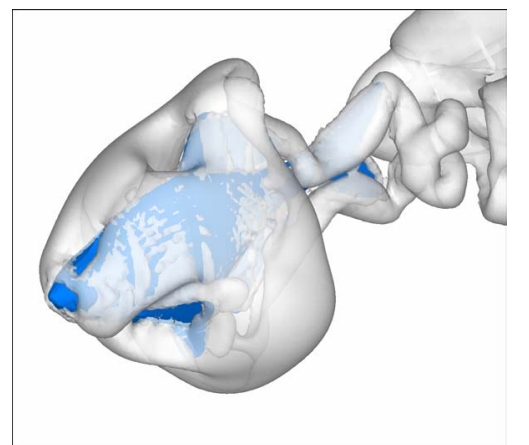

(c) $25 / 100$ frame.

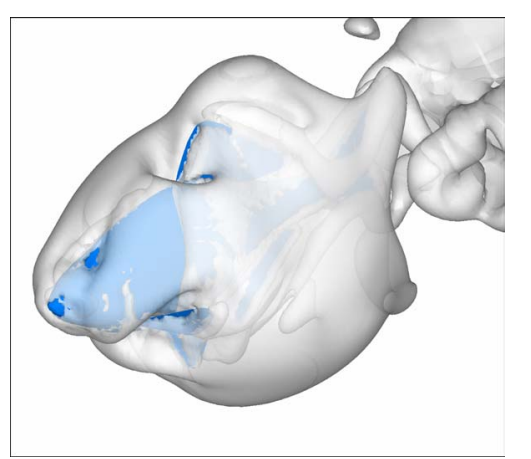

(e) $45 / 100$ frame.

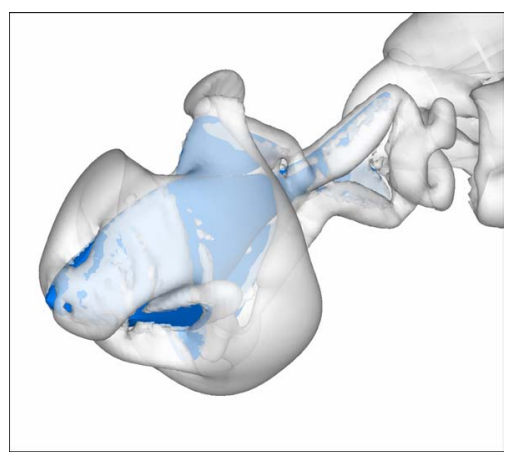

(b) $15 / 100$ frame.

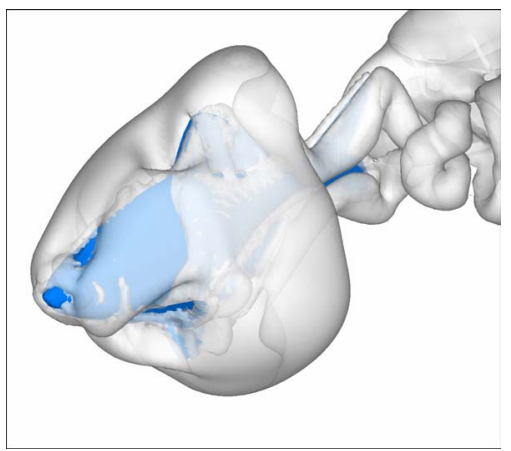

(d) $35 / 100$ frame.

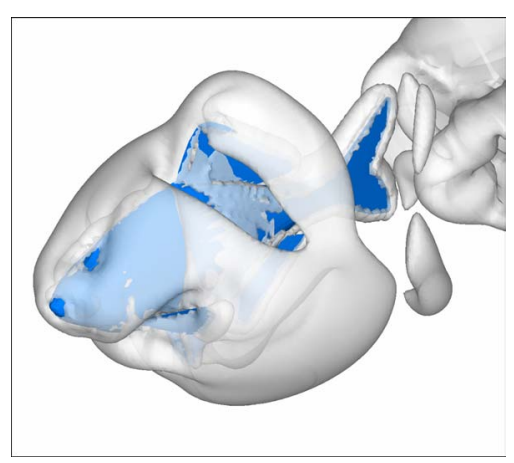

(f) $55 / 100$ frame.

Fig. 17 Iso-surface of second invariant of velocity gradient tensor. 


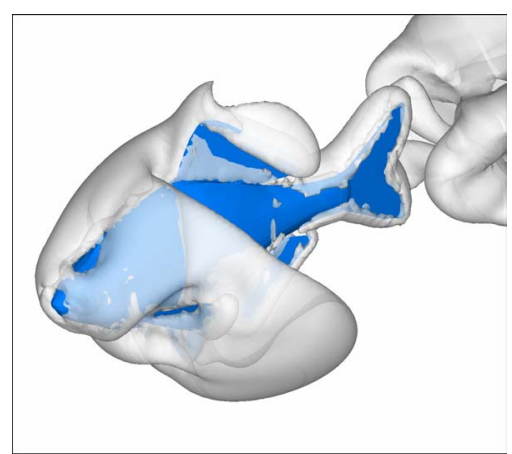

(g) $65 / 100$ frame.

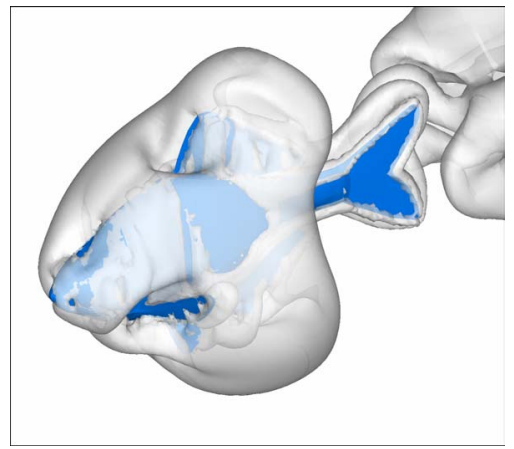

(i) $85 / 100$ frame.

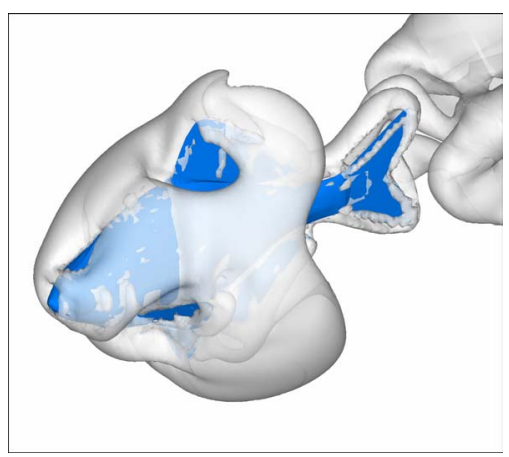

(h) $75 / 100$ frame.

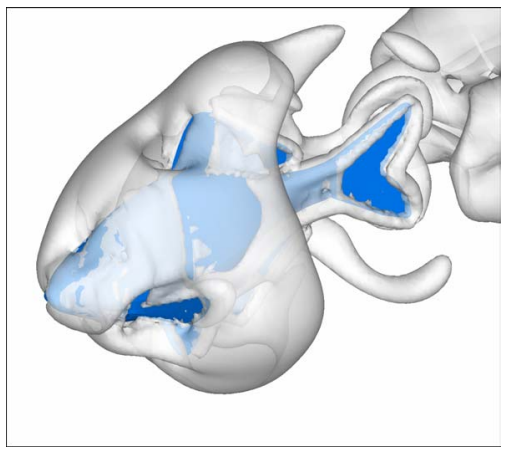

(j) 95/100 frame.

Fig. 17 -Continued.

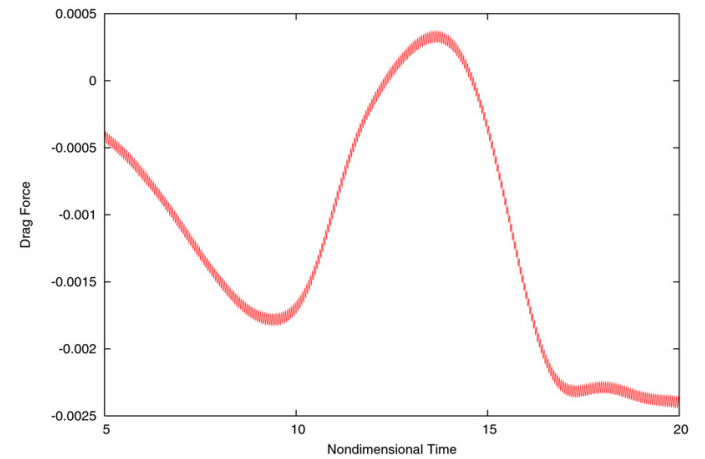

(a) Overall history.

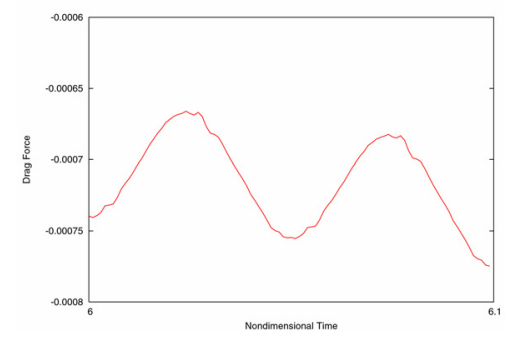

(b) History between $t=6.0$ and 6.1 .

Fig. 18 Time history of non-dimensional drag force.

Figure 18 shows the time history of non-dimensional drag force. The non-dimensional drag force with small fluctuations greatly changes with the time. These small fluctuations are correspondent to the motion with the periodical swimming model. After the initial disturbance comes out from the computational region, the non-dimensional drag force becomes negative value. In comparison with the stationary case, the non-dimensional drag force becomes smaller. Then, it can be confirmed that the some thrust is arising.

\section{Concluding Remarks}

In this paper, the seamless virtual boundary method is applied to the numerical simulation of incompressible flows with complicated geometry and moving boundary. In the flows around a 2D oscillating circular cylinder and a sphere, the present method gives the appropriate flow fields with the smooth pressure distributions and the specified velocity 
conditions on the virtual boundary. For the flow around a stationary fish models, the characteristic surface shape of a fish, i.e., breast fins, abdomen fins, hip fin, back fin and tail fin, can be reappeared by using the triangular polygon. The flows around these fins can be resolved qualitatively. Finally, the swimming fish model is constructed by $2 \mathrm{D}$ rotation on four rotating axes. The flow with the motions of each fin can be clarified and the non-dimensional drag force becomes smaller than the flow around a stationary fish model. Then, it is concluded that the present seamless virtual boundary method is very promising for the numerical simulation of incompressible flows with the complicated geometry and the moving boundary.

\section{References}

(1) E.A. Fadlun, R. Verzicco, P. Orlandi, and J. Mohd-Yosof, Combined immersed-boundary finite-difference methods for three-dimensional complex simulations, J. Comput. Phys., 161, (2000), pp.35-60.

(2) D. Goldstein, R. Handler, and L. Sirovich, Modeling a no-slip flow boundary with an external force field, J. Comput. Phys., 105, (1993), pp.354-366.

(3) H. Nishida, Cartesian grid approach with virtual boundary method and its applications, Notes on Numerical Fluid Mechanics (Springer), 78, (2001), pp.17-32.

(4) E.M. Saiki and S. Biringen, Numerical simulation of a cylinder in uniform flow: application of a virtual boundary method, J. Comput. Phys., 123, (1996), pp.450-465.

(5) M. Tyagi and S. Acharya, Large eddy simulation of turbulent flows in complex and moving rigid geometries using the immersed boundary method, Int. J. Numer. Meth. Fluids, 48, (2005), pp.691-722.

(6) T. Ye, R. Mittal, H.S. Udaykumar, and W. Shyy, An accurate Cartesian grid method for viscous incompressible flows with complex immersed boundaries, J. Comput. Phys., 156, (1999), pp.209-240.

(7) Y.-H. Tseng and J. H. Ferziger, A ghost-cell immersed boundary method for flow in complex geometry, J. Comput. Phys., 192, 593(2003), pp.593-623.

(8) J. Kim, D. Kim, and H. Choi, An immersed-boundary finite-volume method for simulations of flow in complex geometries, J. Comput. Phys., 171, (2001), pp.132-150.

(9) H. Nishida and K. Sasao, Incompressible flow simulations using virtual boundary method with new direct forcing terms estimation, Proc. International Conference on Computational Fluid Dynamics 2006, (2006), pp.185-186.

(10) Y. Morinishi, T.S. Lund, O.V. Vasilyev, and P. Moin, Fully conservative higher order finite difference schemes for incompressible flow, J. Comput. Phys., 143, 90(1998), pp.90-124.

(11) C.H. Rhie and W.L. Chow, Numerical study of the turbulent flow past an airfoil with trailing edge separation, AIAA Journal, 21-11, (1983), pp.1525-1532.

(12) R.A. Renaut, Two-step Runge-Kutta method and hyperbolic partial differential equation, Math. Compt., 55-192, (1990), pp.563-579.

(13) K. Kawakami, H. Nishida, and N. Satofuka, An open boundary condition for the numerical analysis of unsteady incompressible flow using the vorticity-streamfunction formulation, Trans. JSME Ser. B, 60-574, (1994), pp.1891-1896. (in Japanese)

(14) G. Wu, B. Piao, and S. Kuroda, Development of cut cell method for incompressible fluid flows, Proc. 18th Symp. on Computational Fluid Dynamics, D9-2 (CD-ROM), (2004), pp.1-5. (in Japanese)

(15) S. Shirayama, Flow past a sphere: topological transitions of the vorticity field, AIAA Journal, 30-2, (1992), pp.349-358. 